\title{
A HOMOGENIZATION SAMPLING PROCEDURE FOR CALCULATING TRABECULAR BONE EFFECTIVE STIFFNESS AND TISSUE LEVEL STRESS
}

\author{
S. J. Hollister, ${ }^{*}$ J. M. BRenNan $\dagger$ and N. KikUChI
}

*Orthopaedic Research Laboratories, Section of Orthopaedic Surgery, University of Michigan, Ann Arbor, MI 48109, U.S.A.; and †Computational Mechanics Laboratory, Department of Mechanical Engineering and Applied Mechanics, University of Michigan, Ann Arbor, MI 48109, U.S.A.

\begin{abstract}
A homogenization sampling procedure is introduced which allows computation of effective trabecular bone stiffness and individual trabecula level stress based on precise models of trabecular bone architecture. Three-dimensional digitized images of 53 trabecular bone specimens with a resolution of $50 \mu \mathrm{m}$ per voxel were directly converted into three-dimensional finite element meshes by making each voxel an 8node isoparametric brick element. Owing to the large mesh of 8000 elements, an element-by-element preconditioned conjugate gradient (EBEPCG) program was written to solve the local homogenization finite element equations. Predicted effective stiffness measures correlated well with experimental results $\left(R^{2}>0.73\right)$. The predicted effective stiffnesses tended to under estimate the experimental values. Average absolute errors in effective stiffness estimates ranged between 31 and $38 \%$ for the sampling procedure compared to a range $49-150 \%$ for a regression fit to volume fraction squared. Trabecula level stress ranged between -200 and +300 times that predicted by analyzing trabecular bone as a continuum. Both tensile and compressive tissue stresses were engendered by a continuum compressive stress. Trabecula level strain energy density (SED) ranged between 0 and 100 times the continuum SED value for two trabecular specimens. In conclusion, the homogenization sampling procedure consistently predicted the influence of trabecular bone architecture on effective stiffness. It can also provide trabecular tissue stress and strain estimates for arbitrary global loading of whole bones. Tissue stresses and strains showed large variations compared to corresponding continuum level quantities.
\end{abstract}

\section{NOMENCLATURE}

\begin{tabular}{|c|c|}
\hline C & $\begin{array}{l}\text { tissue stiffness, i.e. stiffness of an individual } \\
\text { trabecula }\end{array}$ \\
\hline$C_{0}$ & $\begin{array}{l}\text { effective stiffness, i.e. stiffness of a trabecular } \\
\text { bone cube }\end{array}$ \\
\hline $\begin{array}{l}\text { effective or } \\
\text { global }\end{array}$ & $\begin{array}{l}\text { quantities (stress, strain, stiffness) defined on a } \\
\text { continuum level for trabecular bone, greater } \\
\text { than } 3-5 \text { intertrabecular lengths (Harrigan } \\
\text { et al., 1988) }\end{array}$ \\
\hline $\begin{array}{l}\text { tissue or } \\
\text { local }\end{array}$ & $\begin{array}{l}\text { quantities (stress, strain, stiffness) defined on } \\
\text { the trabecular tissue level }\end{array}$ \\
\hline$\eta$ & $\begin{array}{l}\text { ratio of the characteristic microstructure } \\
\text { length to the characteristic continuum length }\end{array}$ \\
\hline$\varepsilon_{0}$ & effective strain \\
\hline$\varepsilon$ & tissue strain \\
\hline$\sigma_{0}$ & effective stress \\
\hline$\sigma$ & tissue stress. \\
\hline
\end{tabular}

\section{INTRODUCTION}

Trabecular bone is a porous material containing multiple, hierarchical structural levels. This hierarchi-

Received in final form 6 August 1993.

Address correspondence to: Scott J. Hollister, Orthopaedic Research Laboratories, University of Michigan, Rm. G0161400 N. Ingalls Building, Ann Arbor, MI 48109-0486, U.S.A. Tel. (313) 763-9674.

+Current address: Altair Engineering Inc., 3150 Livernois, Suite 270, Troy, MI 48083, U.S.A.

This paper was presented in part at the International Symposium on Computer Methods in Biomechanics and Biomedical Engineering at Swansea, Wales, 5-7 May 1992. cal structural organization determines two important aspects of trabecular bone mechanics and adaptation. First, the structural organization determines the effective stiffness of trabecular bone (Cowin, 1985; Goldstein, 1987; Goulet et al., 1989; Snyder et al., 1989; Turner et al., 1988). Predicting the influence of bone architectural changes on effective stiffness and strength is important for understanding age related bone fragility (Kleerekoper et al., 1985). Second, the structural organization determines how load is transferred to the osteocyte and bone lining cell level. Understanding bone adaptation mechanisms may ultimately require relating stress and strain estimates at the cell level to bone cell activity.

Determining effective stiffness and quantifying microstructural level stresses are two aspects of the same problem. If the stresses at each microstructural level are calculated correctly, then the effective stiffness is accurately predicted because it relates average microstructural stress to strain. Since it is impossible to model each microstructural component of large composite structures, effective stiffness and microstructural stresses are generally approximated using a representative volume element (RVE) approach (Hashin, 1983). The RVE approach consists of analyzing a small section of composite material under assumed boundary conditions, generally, uniform traction or displacement. This analysis provides the relationship between the effective or global strains, $\varepsilon_{0}$, and the microstructural or local strains $\varepsilon$. It also provides the effective stiffness $C_{0}$ which relates the 
effective strain $\varepsilon_{0}$ to the effective stress $\sigma_{0}$. However, RVE analyses, can only provide upper or lower effective stiffness bounds because the precise in situ RVE boundary conditions are not known and must be assumed (Hashin, 1983; Hill, 1963). The familiar Voight and Reuss models are the extreme bounds on RVE stiffness estimates (Hill, 1963).

Trabecular bone microstructural analyses have previously analyzed idealized models using the RVE concept (Beaupré and Hayes, 1985; Gibson, 1985; Pugh et al., 1973; Williams and Lewis, 1982). Although no statistical correlations between experimental and predicted results were reported, it was noted in many cases that the predicted stiffness was greater than the experimental. This overprediction may have resulted from a number of factors including assumptions about trabecular tissue stiffness, idealization of the complex trabecular architecture, and RVE boundary condition assumptions. Beaupre and Hayes (1985) noted that two different displacement RVE boundary conditions gave two different estimates of effective stiffness. These results are consistent with the upper and lower bounding principles of general composite mechanics (Hashin, 1983; Hill, 1963).

Recently, homogenization theory has been applied to analyze both trabecular and cortical bone mechanics (Crolet et al., 1988; Hollister et al., 1991). Although homogenization theory calculates the same quantities as traditional RVE approaches, its formulation is quite different leading to different results (Hollister and Kikuchi, 1992). The periodic boundary displacement assumptions of homogenization theory will give effective stiffness estimates between the upper and lower bounds given by assumed boundary displacements and tractions, respectively (Suquet, 1987). Microstructural stress error bounds for homogenization analysis of periodic structures have been derived (Bakhvalov and Panasenko, 1989). However, Hollister et al. (1991) found that trabecular bone effective stiffness measures calculated by homogenization analysis of idealized models were inconsistent with experimental results. The paper presents and provides initial validation of a homogenization procedure for calculating effective stiffness and tissue level stress directly from precise models of trabecular bone architecture.

\section{METHODS}

The homogenization sampling procedure entails homogenization analysis of selected trabecular bone architecture samples from a large region of trabecular bone. Each sample is one precise microstructural model of trabecular bone architecture based on a three-dimensional digitized image. The key components of the procedure are (1) the homogenization theory formulation, (2) the construction of finite element models from three-dimensional digitized image of trabecular bone based on samples throughout the specimen volume, and (3) the finite element EBEPCG program used to solve the homogenization equations.
The governing equations of homogenization theory used in the sampling procedure are presented briefly. Formulation of homogenization theory and its initial use for trabecular bone analysis may be found in Hollister et al.(1991). More mathcmatical descriptions of homogenization theory may be found in Bakhvalov and Panasenko (1989), Lions (1981), Sanchez-Palencia (1980), and Suquet (1987).

Homogenization theory is based on three fundamental premises. First, the total displacement of a microstructured material is represented by an asymptotic expansion:

$$
\begin{aligned}
u_{\eta i}\left(x_{i}, y_{i}\right)= & u_{0 i}\left(x_{i}\right)+\eta u_{1 i}\left(x_{i}, y_{i}\right) \\
& +\eta^{2} u_{2 i}\left(x_{i}, y_{i}\right)+\cdots,
\end{aligned}
$$

where $u_{\eta i}$ is the total displacement, $u_{0 i}$ is the effective or continuum level displacement, $u_{n i}$ are perturbations in the displacement due to the microstructure, $x_{i}$ are continuum level coordinates, $y_{i}$ are microstructural level coordinates, and $\eta$ is the ratio of the microstructural scale to the continuum scale. Second, it is assumed that quantities on the microstructural level vary $1 / \eta$ times faster than continuum or effective level quantities. Since $\eta$ is always less than one, quantities like stress and strain fluctuate more rapidly on the microstructural scale. In the formulation, $\eta$ does not appear in the 0th order approximation (see thc appendix of Hollister et al., 1991) and it is not assigned an explicit value. However, it is known that for periodic materials the error decreases as $\eta$ decreases (Bakhvalov and Panasenko, 1989; Hollister and Kikuchi, 1992). Third, it is assumed that the displacement and strain fields are periodic functions in $y_{i^{-}}$

Based on the three premises, substitution of the asymptotic expansion into the standard weak form of the linear elastic equilibrium equation yields one set of microstructural equilibrium equations and one continuum or effective level equilibrium equation. The microstructural equilibrium equations are

$$
\begin{aligned}
& \begin{aligned}
\int_{\Omega_{y}}\left\{\varepsilon_{1}(v)\right\}^{\mathrm{T}}[C]\left\{\varepsilon_{1}^{* k}\left(u^{*}\right)\right\} \mathrm{d} \Omega_{y} \\
=\int_{\Omega_{y}}\left\{\varepsilon_{1}(v)\right\}^{\mathrm{T}}\left\{C^{k}\right\} \mathrm{d} \Omega_{y},
\end{aligned} \\
& \varepsilon_{1}^{* k} \text { periodic, } k=1-6,
\end{aligned}
$$

where $\left\{\varepsilon_{1}(v)\right\}$ is the virtual strain vector, $[C]$ is the trabecular tissue stiffness, $\left\{\varepsilon_{1}^{* k}\right\}$ is denoted as the microstructural fluctuating strain vector, and $\left\{C^{k}\right\}$ is the $k$ th column of the tissue stiffness matrix. Equation 2 is solved six times for three-dimensional problems, once for each column of $[C]$. Physically this amounts to loading the microstructure with a volume stress $\left\{C^{k}\right\}$ which results when the $k$ th unit strain vector is multiplied by the stiffness matrix and calculating the displacements under periodic boundary constraints. The solution to equation (2) yields the local structure matrix (Hollister et al., 1991) which relates micro- 
structural to continuum level strains:

$$
\{\varepsilon\}=[M]\left\{\varepsilon_{0}\right\},
$$

where $\{\varepsilon\}$ is the microstructural or tissue level strain, $[M]$ is the local structure matrix, and $\left\{\varepsilon_{0}\right\}$ is the continuum level strain. The continuum or effective stiffness may then be calculated by

$$
\left[C_{0}\right]=\frac{1}{\left|V_{y}\right|} \int_{\Omega_{y}}[C][M] \mathrm{d} \Omega_{y},
$$

where $\left[C_{0}\right]$ is the effective stiffness, $[C]$ is the trabecular tissue stiffness, $[M]$ is the local structure matrix, and $V_{y}$ is the total volume of the microstructure. The macroscopic equilibrium equations can then be written as

$$
\int_{\Omega_{y}}\left\{\varepsilon_{0}(v)\right\}^{\mathbf{T}}\left[C_{0}\right]\left\{\varepsilon_{0}\left(u_{0}\right)\right\} \mathrm{d} \Omega_{x}=\int_{\Gamma_{x}}\{v\}^{\mathbf{T}}\{t\} \mathrm{d} \Gamma_{x}
$$

where $v$ is the virtual displacement and $t$ is an applied traction to the global body. A chart illustrating the microstructural to continuum analysis flow is shown in Fig. 1. Homogenization theory can be used to determine both the dependence of effective stiffness on microstructure [equation (4)] and the transfer of load from the whole bone level to the bone microstructure [equation (3)].

Precise finite element models of trabecular bone architecture were created from three-dimensional digitized images produced by a microcomputed tomography (micro-CT) scanner (Feldkamp et al., 1989; Kuhn et al., 1990). Goulet et al. (1989) have compiled threedimensional digitized image files for $1048 \mathrm{~mm}$ cube specimens of trabecular bone with a resolution of $50 \mu \mathrm{m}$ per voxel. A pre-processing program was written to convert directly each voxel into a three-dimensional 8-noded isoparametric brick element from the image files created by Goulet and co-workers. Each element was assigned either trabecular tissue properties (isotropic; $E=5 \mathrm{GPa}, v=0.3$; see Choi et al., 1990; Mente and Lewis, 1989) or compliant marrow properties (isotropic, $E=0.0005 \mathrm{GPa}, v=0.0$ ). The trabecular tissue and marrow were assumed to be perfectly bonded to each other. The pre-processing program allows the user to select any region of the threedimensional digitized image and convert this into a three-dimensional finite element mesh for the sampling analysis (Fig. 2). An advantage of making each voxel an element is that all elements have the same geometry and element stiffness matrices are only developed for each separate material. For the homogenization sampling analysis, one element stiffness matrix each is developed for trabecular tissue and marrow.

A unique requirement of homogenization analysis is that displacements at one microstructural model boundary must equal displacements at the corresponding location on the opposite boundary. This was previously accomplished (Hollister et al., 1991) using a penalty method. The topology of the digitized image model allows for another method of enforcing periodicity. In this case, corresponding nodes on opposite boundaries are numbered the same to ensure that displacements will be equal (Zienkiewicz and Scott, 1972). A special algorithm was written in the preprocessing routine to define the element connectivity with equivalent node numbering on opposite boundaries. In summary, the pre-processing program develops the element connectivity, assigns either trabecular tissue or bone marrow properties to each element based on the image voxel density, calculates the bone and marrow element stiffness matrices, and calculates element load vectors corresponding to the right-hand side of equation (2).

The regular element structuring of the digitized image mesh is well suited for the use of an iterative

\section{Homogenization Procedure}

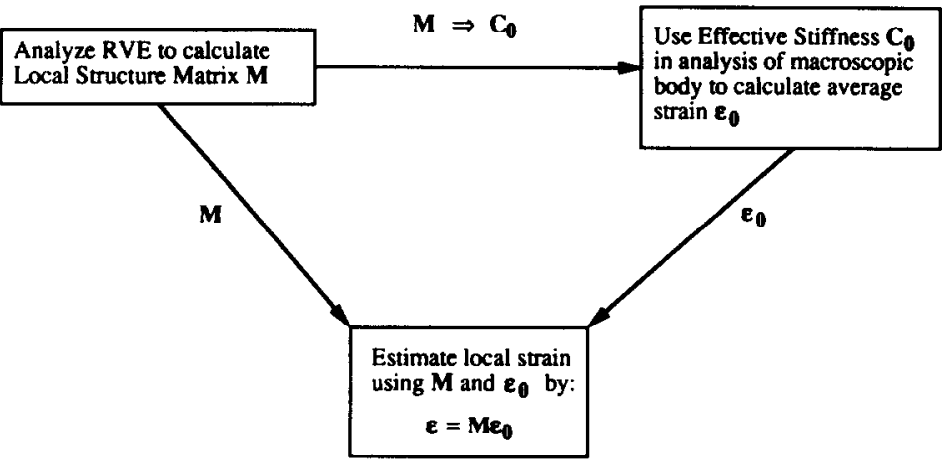

Fig. 1. Schematic of the homogenization RVE analysis procedure. The RVE or trabecular bone microstructure model is first analyzed to calculate the local structure matrix $M$ from which the effective stiffness matrix $C_{0}$ is then calculated. This effective stiffness matrix is then input into a global model (i.e. of a whole joint) to calculate the effective strain distribution, $\varepsilon_{0}$. The effective strain $\varepsilon_{0}$ is then post-processed with the local structure matrix $M$ in a given continuum region to calculate the tissue or local strain distribution, $\varepsilon$, within that region. 


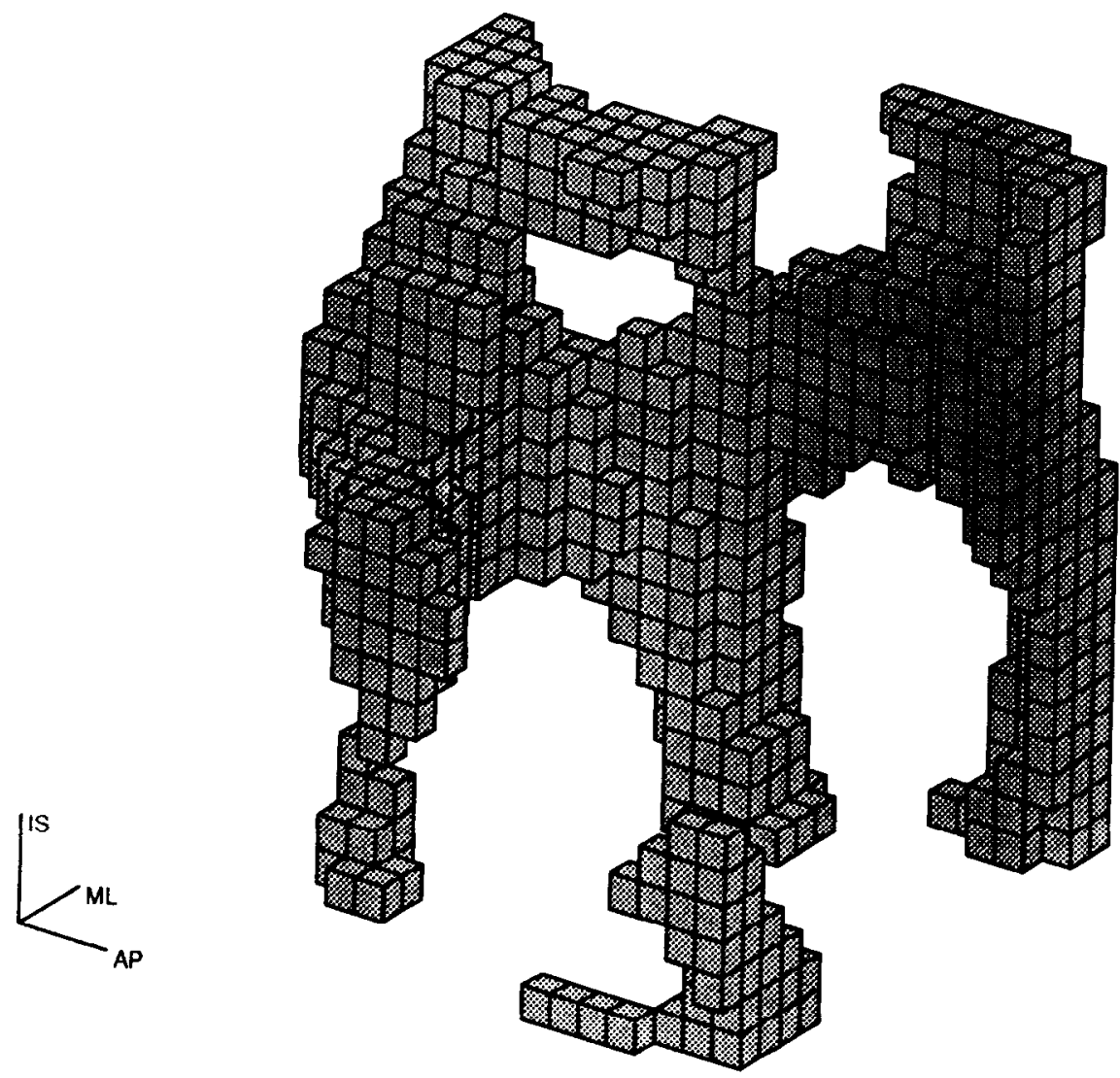

Fig. 2. Three-dimensional finite element mesh from a $1 \mathrm{~mm}^{3}$ volume of lumbar spine trabecular bone (bone elements only are shown). The mesh was constructed by converting each voxel from a three-dimensional digitized image of trabecular bone into an 8-node isoparametric brick element. Each element was then assigned either bone or marrow mechanical properties (marrow elements are shown). The pre-processing program allows the user to select the size and location of regions from the digitized image to convert into a finite element mesh.

equation solver called the element-by-element preconditioned conjugate gradient (EBEPCG) method. Since only matrix vector multiplies are necessary for solving the finite element equations (Ferencz, 1990; Hughes et al., 1983), the global stiffness matrix is never assembled. In the EBEPCG method, a residual is defined as the stiffness matrix displacement vector product minus the load vector. Only the product of the stiffness matrix and the displacement vector is assembled into a global vector (Carey and Jiang, 1986). The iteration continues until the ratio of the current residual vector norm to the initial residual vector norm is less than a user defined tolerance, taken here to be $1.0 \times 10^{-4}$. The initial use of the EBEPCG technique for biomechanics were presented by Fyhrie (1986). Fyhrie and co-workers (1992) also applied the digitized image meshing technique with EBEPCG to perform standard finite element analysis of trabecular bone microstructure using the finite element code NIKE3D.

A special processing program based on the EBEPCG technique was written to solve the homogenization microstructural equilibrium equation [equation (2)]. This program uses the stiffness matrix diagonal for pre-conditioning, commonly known as Jacobi preconditioning (Carey and Jiang, 1986). The finite element processing program reads in the element connectivity, material property identification numbers (ids), element stiffness matrices, and element load vectors output by the pre-processing program. It then solves equation (2) six times, once for each column $\left\{C^{k}\right\}$ of the tissue stiffness matrix. For this study, each $1 \mathrm{~mm}^{3}$ sample contained 8000 solid elements which generally required 2-4 h to solve all six cases on a Sun Sparc 2 (Sun Microsystems) workstation. In summary, the homogenization EBEPCG processing program solves equation (2), recovers the local structure matrix at the element centroids, and calculates the effective trabecular stiffness matrix [equation (4)].

Although the digitized image based mesh offers substantial computational advantages and makes it possible to solve large problems $(>8000$ solid elements) relatively quickly, it introduces sharp corner boundaries between bone and marrow into the discretization. These corners may introduce numerical artifacts into both the effective stiffness and tissue level stress calculations. To estimate the size of these artifacts, two smooth meshes containing a compliant 

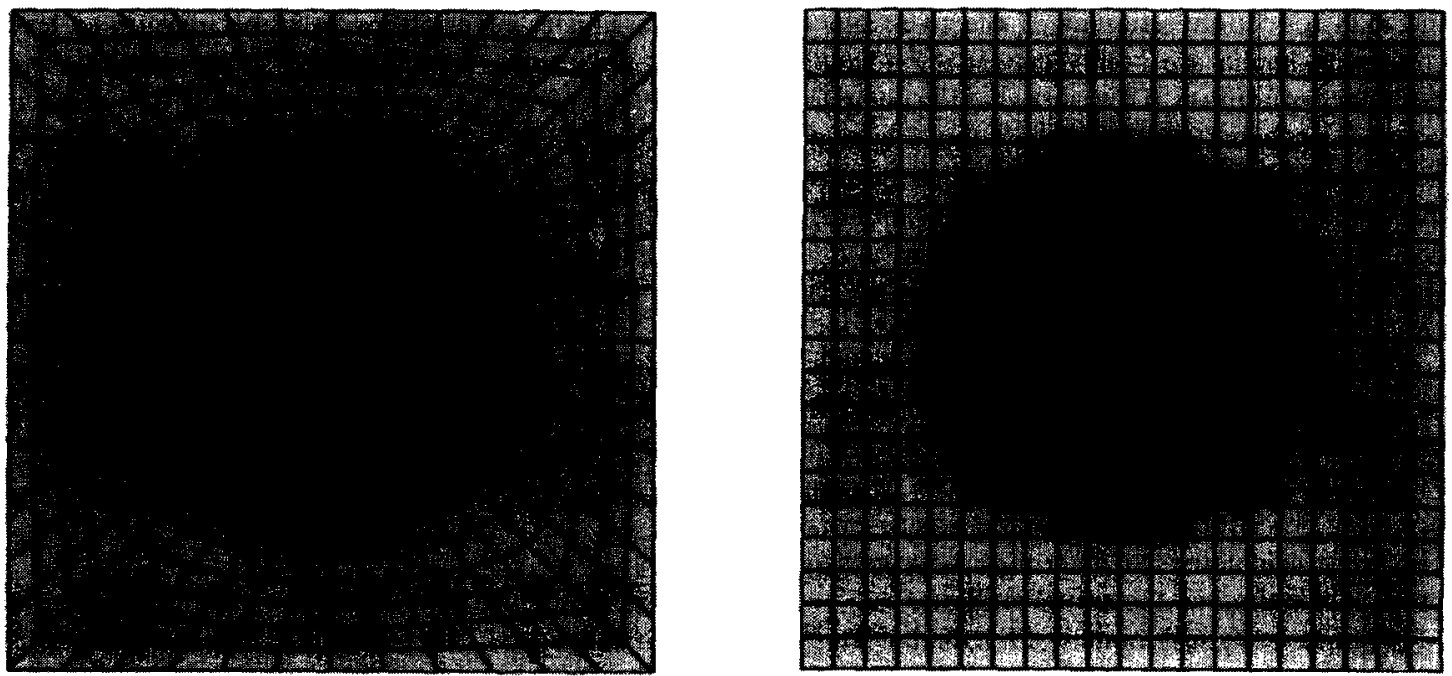

Fig. 3. Smoothed (left) and digitized (right) meshes of bone tissue material with marrow material in the center. The struts are $200 \mu \mathrm{m}$ thick. These meshes were used to assess the affect of the digitized mesh on the calculated effective stiffness. The two meshes have equivalent volume fractions of bone (light grey) and marrow (black). The digitized mesh has a pixel resolution of $50 \mu \mathrm{m}$, the same resolution of the threedimensional digitized images of trabecular bone.
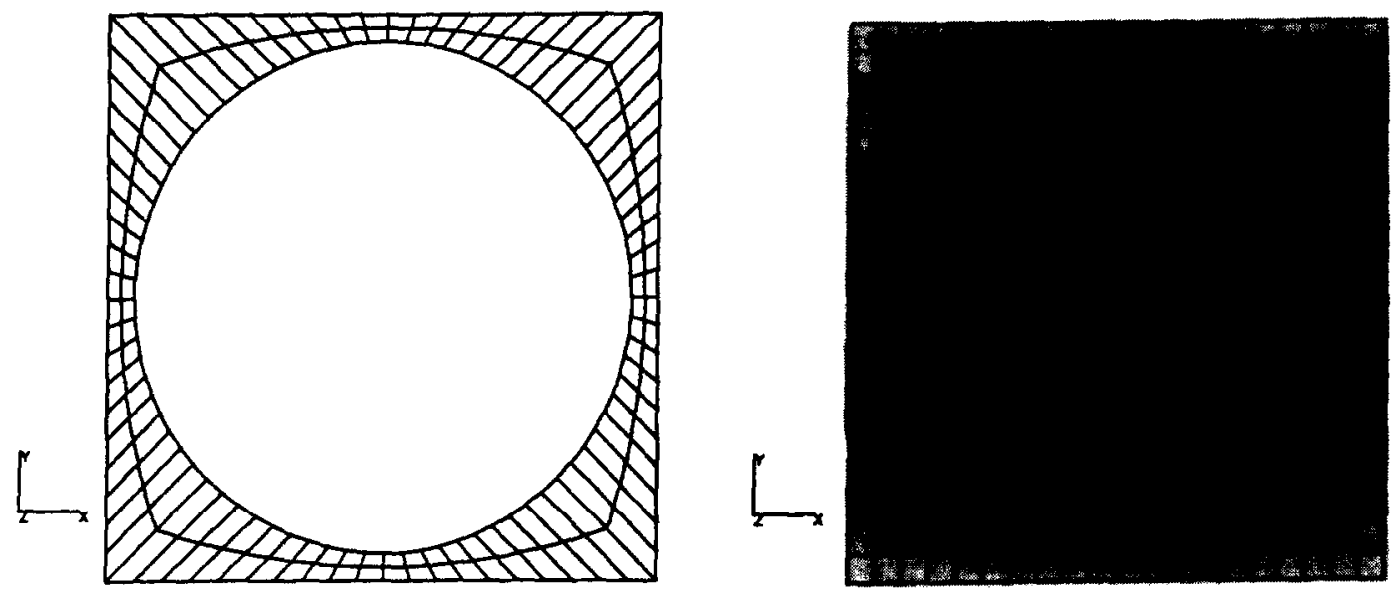

Fig. 4. Smoothed (left) and digitized (right) meshes of bone tissue material with marrow material in the center. The struts in this case are $50 \mu \mathrm{m}$ thick. These meshes were used to assess the affect of the digitized mesh on the calculated effective stiffness. The two meshes have equivalent volume fractions of bone (light grey) and marrow (black). The digitized mesh has a pixel resolution of $50 \mu \mathrm{m}$, the same resolution of the three-dimensional digitized images of trabecular bone.

material center and outer struts similar in thickness to one trabeculae $(200 \mu \mathrm{m}$, Fig. $3 ; 50 \mu \mathrm{m}$, Fig. 4) were analyzed using a two-dimensional homogenization code (Hollister and Kikuchi, 1992). The same structures were also analyzed using digital image based meshes (200 $\mu \mathrm{m}$; Fig. 3; $50 \mu \mathrm{m}$, Fig. 4). The digital image based mesh was analyzed using a two-dimensional version of the homogenization EBEPCG program. The effective stiffness calculated using each approach were then compared.

Fifty-three $8 \times 8 \times 8 \mathrm{~mm}$ cube trabecular bone specimens experimentally tested by Ciarelli et al. (1991) and imaged by Goulet et al. (1994) were analyzed using the sampling procedure to verify the stiffness estimates. These specimens were from the lumbar spine, proximal femur, proximal tibia, iliac crest, distal femur, proximal humerus, and distal radius of four cadavers. Twenty-seven $1 \mathrm{~mm}^{3}$ equally spaced samples were analyzed for each specimen, giving a total of 1431 analyses with 8000 solid elements each. The effective stiffness matrices from each of the 27 samples were then averaged to give one complete anisotropic stiffness matrix for the whole specimen. Ciarelli et al. reported three stiffness values in the Anterior-Posterior (AP), Medial-Lateral (ML), and Inferior-Superior (IS) anatomic orientations. These stifiness values were the ratio of the average stress in the testing direction to the average strain. As discussed 
in the introduction, estimates of effective stiffness for composite media are highly dependent on assumed boundary conditions. To ensure that the comparisons between predicted and experimental measures of stiffness were consistent, an $8 \mathrm{~mm}$ block with the anisotropic stiffness matrix calculated from the sampling procedure was analyzed under displacement conditions similar to those applied experimentally by Ciarelli et al. (1991). The ratio of average stress to average strain in the AP, ML, and IS directions was then calculated. Linear regressions were used to compare predicted to experimental values. In addition, the average absolute percentage differences between the sampling procedure predictions and experimental results were calculated to determine how closely the procedure estimated the actual stiffness.

Previous analytical models of trabecular bone have predicted trabecular bone effective stiffness based on assumptions that trabeculae undergo bending or axial deformation (Gibson, 1985). These analytical models predict that trabecular bone effective stiffness will be related to the solid volume fraction squared for open cell cubic models and to the solid volume fraction for hexagonal open celled models. Specifically, the effective modulus should be equal to the trabecular tissue modulus multiplied by a constant and the volume fraction raised to a given power as $E_{0}=C^{*} E^{*} V_{\mathrm{f}}^{n}$, where $E_{0}$ is the effective modulus, $C$ is a constant, $E$ is the tissue modulus, $V_{\mathrm{f}}$ is the volume fraction, and $n$ is an integer power. To compare the sampling procedure with these analytical models, the assumed tissue modulus of $5 \mathrm{GPa}$ was multiplied by the volume fraction squared and the volume fraction for each of the 53 specimens. The proportionality constant was taken to be 1 in each case. $A$ linear regression was used to compare predictions of the analytical models to the experimental results. The average absolute percentage differences between the analytical predictions and experimental results were also calculated.
To illustrate variations in trabecular tissue level quantities, tissue level stress and strain energy density were calculated for two samples from two different specimens, one from the lumbar spine and one from the proximal tibia. For each specimen, the average strain corresponding to the experimentally determined continuum failure stress (Ciarelli et al., 1991) was used to calculate the tissue level strain distribution using equation (3). The tissue level stress and strain energy density (SED) distribution were then calculated from the tissue strain. The ratio of the tissue SED to the continuum SED was also calculated.

\section{RESULTS}

The mesh singularities resulting from the digitized mesh had a small effect on the effective stiffness results for the structure with $200 \mu \mathrm{m}$ thick struts. Effective elastic constants calculated using the smooth mesh were $E=2576.4 \mathrm{MPa}, G=640.4, v=0.235$. Effective elastic constants calculated using the digitized mesh were $E=2524.2 \mathrm{MPa}, G=610.9, v=0.234$. The percentage differences between effective elastic constants calculated using the two meshes were $2 \%$ for Young's modulus, $4.6 \%$ for the shear modulus, and $0.4 \%$ for Poisson's ratio. For the structure with $50 \mu \mathrm{m}$ thick struts, the effective constants resulting from the smooth mesh analysis were $E=913.1 \mathrm{MPa}, G$ $=54.1 \mathrm{GPa}$, and $v=0.086$. Results from the digitized mesh were $E=844.5 \mathrm{MPa}, G=39.2 \mathrm{GPa}$, and $v$ $=0.090$. Percentage differences between the two meshes were $7.5 \%$ for Young's modulus, $27.5 \%$ for the shear modulus, and $4.7 \%$ for Poisson's ratio.

Predicted stiffness values showed consistent agreement with experimental results for the 53 specimens analyzed (Table 1). Coefficients of determination $\left(K^{2}\right)$ values were all greater than 0.73 . The average absolute percentage differences between predicted and experimental results were between 31 and $38 \%$ (Table 2).

Table 1. Linear regression between experimental and predicted stiffness values for the sampling procedure. Coefficients of the regression equation Experimental $=A+B^{*}$ Predicted are presented along with adjusted $R^{2}, p$ values, and standard error of estimate. $A P=$ anterior-posterior, $M L=$ medial-lateral, IS $=$ inferior-superior

\begin{tabular}{lrcccccc}
\hline $\begin{array}{l}\text { Stiffness } \\
\text { direction }\end{array}$ & A & B & Adjusted $R^{2}$ & $p$ & $n$ & $\begin{array}{c}\text { Standard error } \\
\text { of estimate }\end{array}$ & $\begin{array}{c}\text { Modulus } \\
\text { range (MPa) }\end{array}$ \\
\hline AP & -46.5 & 1.58 & 0.76 & $<0.01$ & 53 & 115.5 & $6-1524$ \\
ML & 8.6 & 1.35 & 0.76 & $<0.01$ & 53 & 62.3 & $12-654$ \\
IS & -62.6 & 1.75 & 0.73 & $<0.01$ & 53 & 154.1 & $42-1113$ \\
\hline
\end{tabular}

Table 2. Mean abolute error (disregarding sign) for the three methods of calculating effective stiffness. The homogenization sampling procedure gave much smaller errors than the other two methods

\begin{tabular}{lccc}
\hline Method & AP average error & ML average error & IS average error \\
\hline Homogenization sampling & 37.8 & 31.0 & 36.6 \\
Volume fraction squared & 117.0 & 150.1 & 49.0 \\
Volume fraction & 1105.7 & 1268.0 & 522.9 \\
\hline
\end{tabular}


Table 3. Linear regression between experimental and predicted stiffness values for the volume fraction squared and volume fraction models. Coefficients of the regression equation Experimental $=A+B^{*}$ Predicted are presented along with adjusted $R^{2}, p$ values, and standard error of estimate. AP = anterior-posterior, $\mathrm{ML}=$ medial-lateral, $\mathrm{IS}=$ inferior-superior. The coefficients of determination $\left(R^{2}\right)$ for the analytical models are less than those for the sampling procedure

\begin{tabular}{|c|c|c|c|c|c|c|c|}
\hline $\begin{array}{l}\text { Stiffness } \\
\text { direction }\end{array}$ & A & B & Adjusted $R^{2}$ & $p$ & $n$ & $\begin{array}{l}\text { Standard error } \\
\text { of estimate }\end{array}$ & $\begin{array}{c}\text { Modulus } \\
\text { range (MPa) }\end{array}$ \\
\hline \multicolumn{8}{|c|}{ Volume fraction squared model } \\
\hline AP & -104.5 & 1.23 & 0.55 & $<0.01$ & 53 & 157.9 & $6-1524$ \\
\hline ML & 25.2 & 0.70 & 0.59 & $<0.01$ & 53 & 82.1 & $12-654$ \\
\hline IS & -111.1 & 1.68 & 0.65 & $<0.01$ & 53 & 172.8 & $42-1113$ \\
\hline \multicolumn{8}{|c|}{ Volume fraction model } \\
\hline AP & -348.0 & 0.51 & 0.49 & $<0.01$ & 53 & 167.8 & $6-1524$ \\
\hline ML & -171.7 & 0.30 & 0.56 & $<0.01$ & 53 & 85.2 & $12-654$ \\
\hline IS & -447.5 & 0.70 & 0.59 & $<0.01$ & 53 & 187.5 & $42-1113$ \\
\hline
\end{tabular}

The regression equation coefficients indicated that the predicted stiffness values tended to underestimate the experimental values.

Analytical volume fraction square and volume fraction models showed relatively good correlations with experimental results (Table 3), although these correlations were not as good as those from the sampling procedure. Both these analytical models tended to overestimate the experimental stiffness values. The average absolute errors for the volume fraction squared model were much higher than the sampling procedure (Table 2), ranging between 49 and $150 \%$. The simple volume fraction model greatly overestimated the effective stiffness (Table 2), with average absolute errors greater than $500 \%$.

Tissue level stresses ranged between -200 and +300 times that predicted by analyzing trabecular bone as a continuum. Under a continuum AP compressive stress of $-1.03 \mathrm{MPa}$ the lumbar spine trabecular tissue stresses ranged from $94 \mathrm{MPa}$ in tension to $-350 \mathrm{MPa}$ in compression (Fig. 5). Under a continuum AP compressive stress of $-0.62 \mathrm{MPa}$ the proximal tibia trabecular tissue stresses ranged from $144 \mathrm{MPa}$ in tension to $-40 \mathrm{MPa}$ in compression (Fig. 4). In each case, trabeculae perpendicular to the continuum load experienced tension and compression, probably due to bending, while trabeculae parallel to the continuum load experienced primarily compression, probably due to axial deformation.

Trabecular tissue to continuum SED ratios ranged between 0 and 100 over large regions of both the lumbar spine and proximal tibia specimen. The complete SED ratio for the lumbar spine sample ranged from 0 to 350 (Fig. 5). The complete SED ratio for the proximal tibia sample ranged from 0 to 320 (Fig. 6). Ratios less than one indicated trabeculae which were relatively unloaded for the applied continuum stress. A current technique used to estimate tissue level stresses for remodeling analyses (Beaupré et al., 1990; Carter et al., 1987; Huiskes et al., 1987) is to divide the continuum SED by the volume fraction raised to the first or second power. Dividing the continuum SED by the volume fraction gave a ratio of 6.9 (volume fraction $=0.145$ ) and 9.2 (volume fraction $=0.109$ ) for the lumbar spine and proximal tibia sample, respectively. Dividing the continuum SED by the volume fraction squared gave a ratio of 47.6 and 85.8 for the lumbar spine and proximal tibia sample, respectively. SED ratios for these magnitudes occurred in limited areas of the trabecular tissue.

\section{DISCUSSION}

Calculating trabecular tissue level stress and effective stiffness with known accuracy is a critical step towards understanding how bone cells sense mechanical stimuli and adapt trabecular bone tissue in response. RVE based microstructural analysis is the most feasible approach for analyzing bone micromechanics. RVE based methods, including standard mechanics models, self-consistent methods (Hashin, 1983), and homogenization theory, all provide estimates of effective stiffness and tissue or microstructural level stress. The difference between these approaches results from their intrinsic assumptions, primarily concerning RVE boundary conditions. It is difficult to assess the accuracy of tissue level stress estimates made by RVE methods because there is no gold standard to which the estimates can be compared. However, the effective stiffness measures, can be validated with experimental results. Since the effective stiffness relates average tissue stress and strain, validation of effective stiffness is the first step towards more rigorous validation of tissue stress and strain estimates.

A variant of homogenization theory, denoted as the homogenization sampling procedure, has been developed for analyzing trabecular bone micromechanics. This method, as with all RVE based approaches, makes assumptions concerning the RVE boundary conditions, in this case periodic boundary displacements. Boundary condition assumptions along with trabecular tissue stiffness assumptions based on early experimental results contribute the most to uncertainty in trabecular tissue stress estimates. At this 


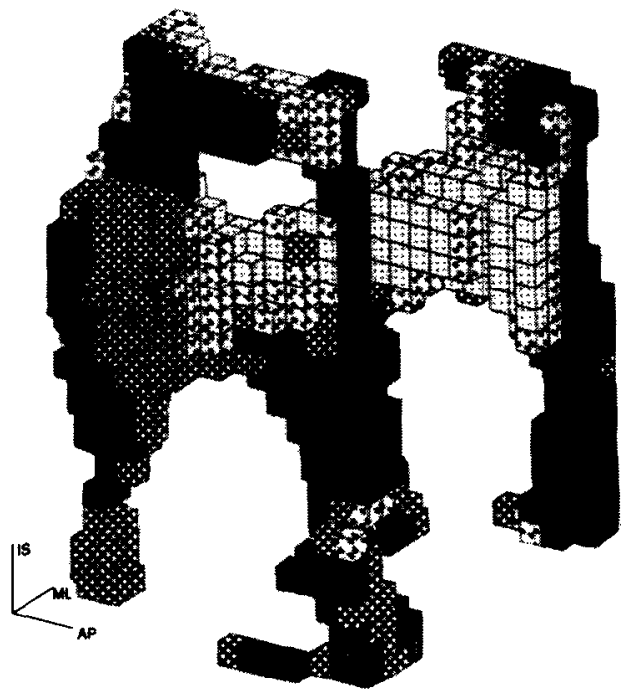

Tissue Z Normal Stress (MPa)

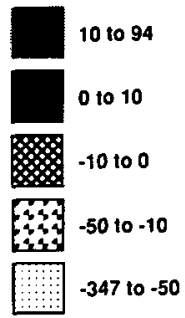

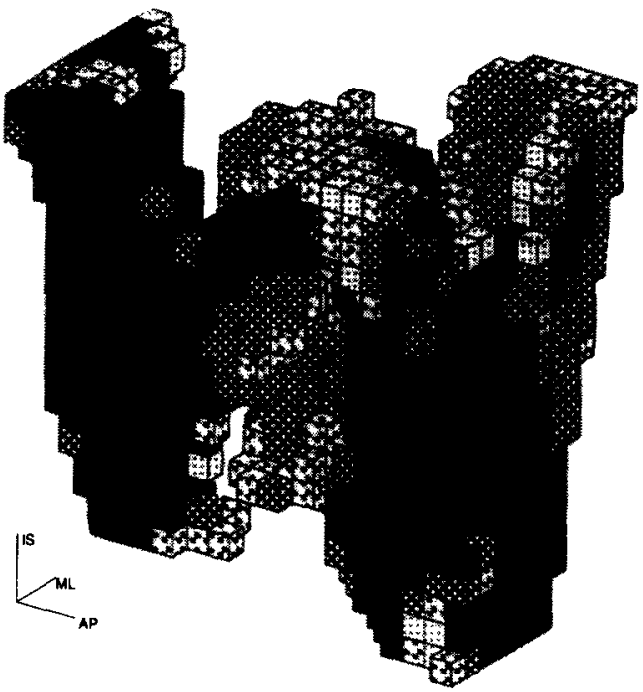

Tissue Z Normal Stress (MPa)

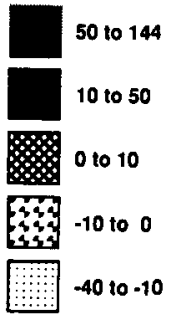

Fig. 5. Distribution of tissue level stress $\sigma_{z z}$ for a $1 \mathrm{~mm}^{3}$ volume of lumbar spine trabecular bone (left) and proximal tibial trabecular bone (right). Continuum level compressive $\sigma_{z z}$ stresses in the AP direction of -1.03 and $-0.62 \mathrm{MPa}$ were applied to the lumbar spine and proximal tibia specimens, respectively. The lumbar spine tissue level stresses ranged from compressive stresses of $-350 \mathrm{MPa}$ to tensile stresses of $94 \mathrm{MPa}$. The proximal tibia tissue level stresses ranged from $-40 \mathrm{MPa}$ in compression to $144 \mathrm{MPa}$ in tension. These results demonstrate the wide variation in tissue level stresses engendered by continuum level stresses. Given the small difference in volume fraction of the two specimens, these results suggest that the distribution of tissue level stresses is as much a function of the trabecular architecture as the bone volume fraction.

point, the uncertainty locally in trabecular tissue stresses has not been quantified. However, correlations with experimental effective stiffness show that mean tissue level stresses can be consistently estimated. A technical limitation of the method is the extensive computational requirements. However, the implementation of EBEPCG allows the method to be run on standard engineering workstations. If tissue level stress and effective stiffness estimates are needed for specific trabecular structures, perhaps for analyzing bone remodeling experimentals, the added computational expenses are appropriate.

The use of homogenization theory for trabecular bone analysis has been deemed inappropriate by some because of the argument that bone is not periodic. However, the periodicity assumption should be viewed in the context that any RVE based method must make assumptions about in situ boundary conditions. Previous RVE analyses of trabecular bone have generally assumed uniform traction or displacement boundary conditions. These assumptions can be shown to give upper and lower bounds on the effective stiffness. The difference between these bounds is substantial for porous materials with low volume fraction like trabecular bone. Both Suquet (1985) and Hollister and Kikuchi (1992) have shown that the periodicity boundary conditions used in homogenization theory are a compromise between applied traction and displacement RVE boundary condition assumptions. Because of this compromise, the periodicity assumption will give an estimate between the upper and lower bounds produced by traditional RVE methods. The homogenization based sampling method produced more accurate trabecular bone effective stiffness estimates than previous RVE based methods.

The use of a digitized mesh presents a trade-off between the ability to construct and analyze numerous complicated three-dimensional meshes containing tens of thousands of elements and the numerical artifacts introduced by the jagged mesh. The results of 


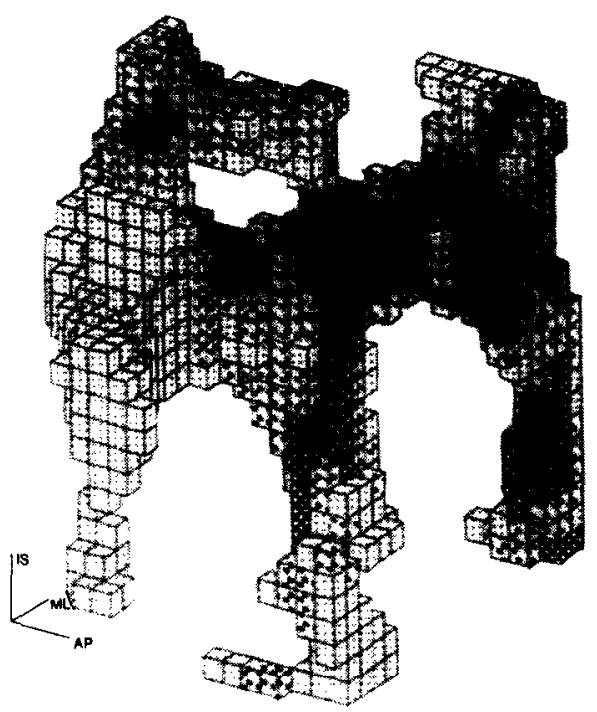

Tissue/Continuum SED Ratio

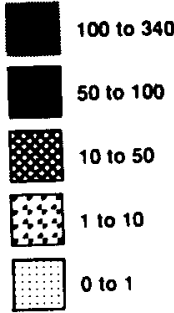

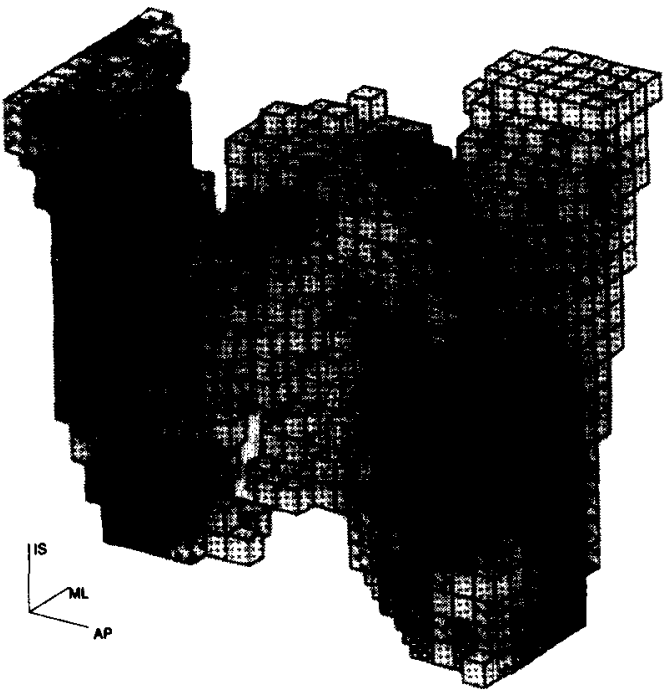

Tissue/Continuum SED Ratio

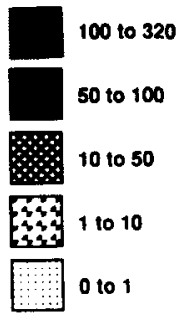

Fig. 6. Tissue/continuum strain energy density (SED) ratios for a $1 \mathrm{~mm}^{3}$ volume of lumbar spine trabecular bone (left) and proximal tibial trabecular bone (right). The majority of the tissue from each specimen shows a ratio between 0 and 100 . Isolated regions show ratios up to 320 . Some of the tissue (areas covered by light dots) has SED ratios less than one indicating that these portions of the trabeculae may be relatively unloaded. These results suggest that dividing the continuum SED by the bone volume fraction is a better reference for predicting bone adaptation than the unmodified continuum SED. However, scaling the continuum SED by the inverse of the bone volume fraction will not predict which portions of the trabeculae are unloaded.

this study would suggest that numerical artifacts may introduce about a $5-10 \%$ error in the effective Young's modulus calculations, even when only one element is present through the thickness. Errors for shear moduli may be higher especially if only one element is present through the thickness of the strut. Although significant, this error is probably much less than errors which could be attributed to the use of an RVE approach and uncertainties in the assumed material properties for individual trabeculae. The errors in local stress calculations introduced by the jagged mesh may be more substantial requiring some numerical smoothing techniques to reduce the artifact. However, Keyak et al. (1992) found a good correlation (regression slope 0.85 ) between cortical bone strains predicted by a CT generated digital image mesh and experimental results. Furthermore, Hollister and Riemer (1993) found that digital image and smooth finite element meshes with the same mesh density produced very similar SED distributions except in a very localized boundary region between dissimilar materials.

Sampling procedure predictions of effective stiffness correlated well with experimental results and had average absolute errors ranging between 31 and $38 \%$. In contrast, average absolute errors for idealized models based on bone volume fraction squared ranged between 49 and $150 \%$ while errors ranged between 522 and $1268 \%$ for linear volume fraction models. Hollister et al. (1991) also found that idealized trabecular bone models did not consistently predict experimental results. The wide variation in trabecular bone architecture may preclude accurate prediction of effective stiffness for specific specimens using idealized models based on assumptions about trabecular deformation. This study suggests that the widely varying architecture and trabecular orientation within even a $1 \mathrm{~mm}$ volume will induce complex trabecular deformations under uniform continuum loads. While idealized microstructural models provide reasonably good 
generalized and qualitative predictions of effective stiffness, computational analysis based on precise trabecular bone architecture models may be necessary to quantitate effective stiffness for specific trabecular bone specimens.

The ability to precisely estimate the effective stiffness of specific trabecular bone architectures is particularly relevant for studies of age related bone fragility. Parfitt and co-workers (Kleerekoper et al., 1985; Parfitt, 1987; Parfitt et al., 1983) have both shown that increased fracture risk with aging is related more to loss of structural elements than uniform thinning of trabeculae in trabecular bone. Mosekilde and coworkers (Mosekilde, 1988, 1990b; Mosekilde and Mosekilde, 1988) have shown loss of trabecular continuity and a preferential loss of horizontal trabeculae in vertebral bodies with age. These specific changes in trabecular bone architecture can be readily incorporated into the homogenization sampling procedure to predict the resulting changes in effective stiffness and strength. Furthermore, it would be possible to predict tissue level stress alterations in remaining trabeculae after loss of trabeculae continuity and structural elements. Tissue level stress estimates could be used to elucidate the relationship between increased stress in vertical trabeculae resulting from horizontal trabecular dropout which has been hypothesized to cause trabecular fracture with microcallus formation (Mosekilde, 1990b).

A striking feature of this study is the large variation in tissue level stresses based on one continuum level stress. The results are consistent with those of Hollister et al. (1991) who found maximum tissue/continuum SED ratios ranging between 60 and 100 for idealized trabecular bone models with volume fractions of 0.10 . The volume fractions of the lumbar spine and proximal tibia specimens were 0.109 and 0.145 , respectively. Despite the small difference in volume fraction, the ratio between the tissue and continuum level $\sigma_{z z}$ stress varied from -91 to 340 for the lumbar spine sample and from -232 to 65 for the proximal tibia sample. The difference between continuum and tissue level stresses may thus depend as much on the specific trabecular architecture as on the bone volume fraction.

Large variations in trabecular tissue stress within a continuum volume may have important ramifications for studies of trabecular bone remodeling. Many previous investigators have recognized that continuum stress measures cannot reflect the wide variation in tissue stresses. Carter et al. (1987) stated "the continuum model for trabecular bone misrepresents the true stress and energy values of the mineralized bone tissue". They proposed dividing the continuum SED by the bone volume fraction to estimate the tissue level SED. Huiskes et al. (1987) also stated that it would be better to use the continuum SED divided by bone volume fraction as an estimate of a tissue level remodeling stimulus than the continuum SED. Wein- ans (1991) utilized this tissue level estimate in later remodeling simulations. Beaupré et al. (1990) proposed a tissue level remodeling stimulus equal to a continuum remodeling stimulus divided by the bone volume fraction squared.

Two observations about tissue level remodeling stimuli and continuum analyses may be made based on tissue level stress estimates from this study. First, it is clear that dividing a continuum level quantity by the bone volume fraction squared or to the first power gives a better estimate of tissue stress states than continuum quantities alone. Second, it is also clear, however, that dividing the continuum stress state by a scalar does not adequately represent the complete range of tissue level stresses. Under the same continuum stress, some trabeculae may be heavily stressed while others may be nearly unloaded, even within a $1 \mathrm{~mm}^{3}$ volume. This tissue level stress pattern may lead to different adaptations of trabeculae. For example, Mosekilde (1990b) noted that unloaded trabeculae were subject to aggressive osteoclastic resorption, while still connected and loaded trabeculae underwent the normal activation-resorptionformation remodeling sequence. Modified continuum level remodeling stimuli would not differentiate between unloaded and loaded trabeculae within small volumes of trabecular bone. Furthermore, tissue level stress estimates showed that trabaculae within a volume subject to a continuum compressive stress experienced both tension and compressive stresses at the tissue level. Some investigators have postulated that bone may remodel differently under tension and compression (Frost, 1986; Fyhrie and Hollister, 1991a,b). Scalar modified continuum stress measures would not be able to predict which trabeculae may be subject to tension or compression.

In conclusion, the homogenization sampling procedure presented here has given estimates of trabecular bone effective stiffness consistent with experimental results. The procedure also can be used to calculate tissue level stresses. Tissue stresses so calculated ranged between -200 and +300 times continuum stresses for two samples of trabecular bone. The current application of the method is to selectively sample a microstructure. However, completely sampling the entire microstructure may yield more accurate results with the trade off being a substantial increase in computing time. Future work should address the accuracy of tissue level stresses and investigate numerical techniques which can estimate and decrease errors in tissue level stresses introduced by the digitized image based mesh.

Acknowledgements - The authors gratefully acknowledge the help of Robert Goulet and Fred Champlain concerning formats of the digital image files. We would also like to thank Dr Steven Goldstein, Robert Goulet, and Mike Ciarelli for input concerning experimental data from their research and Dr David Fyhrie for discussions concerning the EBEPCG 
method. This work was supported by the National Institutes of Health (AR34399 and AR41349).

\section{REFERENCES}

Bakhvalov, N. and Panasenko, G. (1989) Homogenisation: Averaging Processes in Periodic Media. Kluwer, Dordrecht.

Beaupre, G. S. and Hayes, W. C. (1985) Finite element analysis of a three-dimensional open-celled model for trabecular bone. J. biomech. Engng 107, 249-256.

Beaupré, G. S., Orr, T. E. and Carter, D. R. (1990) An approach for time-dependent bone modeling and remodeling-theoretical development. J. Orthop. Res. 8, 651-661.

Carey, G. F. and Jiang, B. N. (1986) Element-by-element linear and nonlinear solution schemes. Comm. appl. Num. Meth. 2, 145-153.

Carter, D. R., Fyhrie, D. P. and Whalen, R. T. (1987) Trabecular bone density and loading history: regulation of connective tissue biology by mechanical energy. $J$. Biomechanics 20, $785-794$.

Choi, K., Kuhn, J. L., Ciarelli, M. J. and Goldstein, S. A. (1990) The elastic modulus of trabecular, subchondral, and cortical bone tissue and the size-dependency of cortical bone modulus. J. Biomechanics 23, 1103-1113.

Ciarelli, M. J., Goldstein, S. A., Kuhn, J. L., Cody, D. D. and Brown, M. B. (1991) Evaluation of orthogonal mechanical properties and density of human trabecular bone from the major metaphyseal regions with materials testing and computed tomography. J. Orthop. Res. 9, 674-682.

Cowin, S. C. (1985) The relationship between the elasticity tensor and the fabric tensor. Mech. Mat. 4, 137-147.

Crolet, J. M., Aoubiza, B. and Meunier, A. (1988) Numerical model of anisotropic elastic properties of osteons. Proc. 12th American Soc. Biomech, pp. 177-178.

Crolet, J. M., Aoubiza, B. and Meunier, A. (1993) Compact bone: numerical simulation of mechanical characteristics. J. Biomechanics 26, 677-689.

Feldkamp, L. A., Goldstein, S. A., Parfitt, A. M., Jesion, G. and Kleerekoper, M. (1989) The direct examinatin of three dimensional bone architecture in vitro by computed tomography. J. Bone Min. Res. 4, 3-11.

Ferencz, R. M. (1990) Element-by-element preconditioning techniques for large-scale, vectorized finite element analysis in nonlinear solid and structural mechanics. Ph.D. dissertation, Stanford University.

Frost, H. M.(1986) Intermediary Organization of the Skeleton, Vol. I. CRC Press, Boca Raton.

Fyhrie, D. P. (1986) Stress analysis and design of proximal femoral prostheses. Ph.D. dissertation, Stanford University.

Fyhrie, D. P., Hamid, M. S., Kuo, R. F. and Lang, S. M. (1992) Direct three-dimensional finite element analysis of human vertebral cancellous bone. Trans. 38th meeting Orthop. Res. Soc. 17, p. 551.

Fyhrie, D. P. and Hollister, S. J. (1991a) A tissue strain remodeling theory for trabecular bone using homogenization theory. Trans. 36th meeting Orthop. Res. Soc. 15, p. 76.

Fyhrie, D. P. and Hollister, S. J. (1991b) Comparison of a trabecular tissue strain remodeling theory to experimental results. Trans. 36th meeting Orthop. Res. Soc., 15, p. 107.

Gibson, L. J. (1985) The mechanical behavior of cancellous bone. J. Biomechanics 18, 317-328.

Goldstein, S. A. (1987) The mechanical properties of trabecular bone: dependence on anatomical location and function. J. Biomechanics 20, 1055-1061.

Goulet, R. W., Feldkamp, L. A., Kubinski, D. J. and Goldstein, S. A. (1989) Predicting the architectural orientation of trabecular bone, Trans. 35th meeting Orthop. Res. Soc. 14, p. 263.

Goulet, R. W., Goldstein, S. A., Ciarelli, M. J., Kuhn, J. L., Brown, M. B. and Feldkamp, L. A. (1994) The relationship between the structural and mechanical properties of trabecular bone. J. Biomechanics 27, 375-389.

Harrigan, T. P., Jasty, M., Mann, R. W. and Harris, W. H. (1988) Limitations of the continuum assumption in cancellous bone. J. Biomechanics 21, 269-275.

Hashin, Z. (1983) Analysis of composite materials-a survey. J. appl. Mech. 50, 481-505.

Hill, R. (1963) Elastic properties of reinforced solids: some theoretical principles. J. Mech. Phys. Solids 11, 357-372. Hollister, S. J., Fyhrie, D. P., Jepsen, K. J. and Goldstein, S. A. (1991) Application of homogenization theory to the study of trabecular bone mechanics. J. Biomechanics 24, 825-839.

Hollister, S. J. and Kikuchi, N. (1992) A comparison of homogenization and standard mechanics analyses for periodic porous composites. Comput. Mech. 10, 73-95.

Hollister, S. J. and Riemer, B. A. (1993) Digital image based finite element analysis for bone microstructure using conjugate gradient and Gaussian filter techniques, Proceedings of the SPIE Mathematical Methods in Medical Imaging II Conference, SPIE Proceedings Vol. 2035, pp. 95-106.

Hughes, T. J. R., Levit, I. and Winget, J. M. (1983) An element-by-element solution algorithm for problems of structural and solid mechanics. Comput. Mech. Appl. Mech. Engng 36, 241-254.

Huiskes, R., Weinans, H., Grootenboer, H. J., Dalstra, M., Fudala, B. and Slooff, T. J. (1987) Adaptive boneremodeling applied to prosthetic-design analysis. $J$. Biomechanics 20, 1135-1150.

Keyak, J. H., Fourkas, M. J., Meagher, J. M. and Skinner, H. B. (1992) Strain gauge verification of an automated method of finite element modeling of bone from CT scan data. Trans. 38th meeting Orthop. Res. Soc., 17, 553.

Kleerekoper, M., Villanueva, A. R., Stanciu, J., Rao, D. S. and Parfitt, A. M. (1985) The role of three-dimensional trabecular microstructure in the pathogenesis of vertebral compression fractures. Calcif. Tissue Int. 37, 594-597.

Kuhn, J. L., Goldstein, S. A., Feldkamp, L. A., Goulet, R. W. and Jesion, G. (1990) Evaluation of a microcomputed tomography system to study trabecular bone structure. J. Orthop. Res. 8, 833-842.

Lions, J. (1981) Some Methods in the Mathematical Analysis of Systems and their Control. Gordon and Breach, New York.

Martin, R. B. and Burr, D. B. (1989) Structure, Function, and Adaptation of Compact Bone. Raven Press, New York.

Mente, P. L. and Lewis, J. L. (1989) Experimental method for the measurement of the elastic modulus of trabecular bone tissue. J. Orthop. Res. 7, 456-461.

Mosekilde, L. (1988) Age-related changes in vertebral trabecular bone architecture-assessed by a new method. Bone 9, 247-250.

Mosekilde, L. (1990a) Age-related loss of vertebral trabecular bone mass and structure-biomechanical consequences. In Biomechanics of Diarthrodial Joints Vol. II. Springer, Berlin.

Mosekilde, L. (1990b) Consequences of the remodelling process for vertebral trabecular bone structure: a scanning electron microscopy study (uncoupling of unloaded structures). Bone and Mineral 10, 13-35.

Mosekilde, L. and Mosekilde, L. (1988) Iliac crest trabecular bone volume as predictor for vertebral compressive strength, ash density and trabecular bone volume in normal individuals. Bone 9, 195-199.

Parfitt, A. M. (1987) Bone remodeling and bone loss: understanding the pathophysiology of osteoporosis. Clin. Obstetrics Gynecology 30, 789-811. 
Parfitt, A. M., Mathews, C. H. E., Villanueva, A. R., Kleerekoper, M., Frame, B. and Rao, D. S. (1983) Relationships between surface, volume, and thickness of iliac trabecular bone in aging and in osteoporosis. J. clin. Invest. 72 , 1396-1409.

Pugh, J. W., Rose, R. M. and Radin, E. L. (1973) A structural model for the mechanical behavior of trabecular bone. $J$. Biomechanics 6, 657-670.

Sanchez-Palencia, E. (1980) Non-Homogeneous Media and Vibration Theory. Springer, Berlin.

Snyder, B. D., Cheal, E. J., Hipp, J. A. and Hayes, W. C. (1989) Anisotropic structure-property relation for trabecular bone. Trans. 35th Orthop. Res. Soc. 14, p. 262.

Suquet, P. M. (1987) Elements of homogenization theory for inelastic solid mechanics. In Homogenization Techniques for Composite Media (Edited by Sanchez-Palencia, E. and Zaoui, A.). Springer, Berlin.

Turner, C. H., Cowin, S. C., Rho, J. Y., Ashman, R. B. and Rice, J. C. (1988) The fabric dependence of the orthotropic elastic constants of cancellous bone. $J$. Biomechanics $\mathbf{2 3}$, $549-561$.

Weinans, H. (1991) Mechanically induced bone adaptations around orthopaedic implants. Ph.D. dissertation, University of Nijmegen.

Williams, J. L. and Lewis, J. L. (1982) Properties and an anisotropic model of cancellous bone from the proximal tibia epiphysis. J. biomech. Engng 104, 50-56.

Zienkiewicz, O. C. and Scott, F. C. (1972) On the principle of repeatability and its application in analysis of turbine and pump impellers. Int. J. Num. Meth. Engng 4, 445-448. 